ELECTRONIC RESEARCH ANNOUNCEMENTS

OF THE AMERICAN MATHEMATICAL SOCIETY

Volume 10, Pages 103-112 (September 27, 2004)

S $1079-6762(04) 00135-0$

\title{
AN UPPER BOUND FOR POSITIVE SOLUTIONS OF THE EQUATION $\Delta u=u^{\alpha}$
}

\author{
S. E. KUZNETSOV
}

(Communicated by Mark Freidlin)

\begin{abstract}
In 2002 Mselati proved that every positive solution of the equation $\Delta u=u^{2}$ in a bounded domain of class $C^{4}$ is the limit of an increasing sequence of moderate solutions. (A solution is called moderate if it is dominated by a harmonic function.) As a part of his proof, he established an upper bound (in terms of the capacity of $K$ ) for solutions vanishing off a compact subset $K$ of $\partial E$. We use a different kind of capacity (we call it the Poisson capacity) and we establish in terms of this capacity an upper bound for solutions of $\Delta u=u^{\alpha}$ with $1<\alpha \leq 2$. This is a part of the program: to classify all positive solutions of this equation.
\end{abstract}

\section{INTRODUCTION}

1.1. Main result. Let $E \subset \mathbb{R}^{d}$ be a bounded smooth domain of class $C^{4}$ in $\mathbb{R}^{d}$. For $x \in E$, we denote by $\rho(x)$ the distance to the boundary $\partial E$ and by $k(x, y)$ the Poisson kernel in $E$ for the Laplacian $\Delta$.

Let $\mathcal{M}(S)$ stand for the set of all finite measures on a measurable space $S$. For every $\nu \in \mathcal{M}(\partial E)$, we denote by $h_{\nu}$ the harmonic function $h_{\nu}(x)=\int_{\partial E} k(x, y) \nu(d y)$.

For every $\alpha>1$ and every Radon measure $m$ on $E$, there exists a Choquet capacity given on compact subsets of $\partial E$ by the formula

$$
\operatorname{Cap}(K)=\sup _{\nu \in \mathcal{P}(K)} \mathcal{E}(\nu)^{-1}
$$

where $\mathcal{P}(K)$ is the set of all probability measures on $K$ and

$$
\mathcal{E}(\nu)=\int_{E} h_{\nu}(x)^{\alpha} m(d x) .
$$

We call Cap the Poisson capacity.

Our goal is to establish the following theorem.

Theorem 1.1. Suppose Cap is the Poisson capacity corresponding to $1<\alpha \leq 2$ and the measure $m(d x)=\rho(x) d x$. Let $K$ be a compact subset of $\partial E$. There exists a constant $C(E)$ depending only on $E$, such that, for every compact $K \subset \partial E$ and

Received by the editors April 5, 2004.

2000 Mathematics Subject Classification. Primary 35J15; Secondary 35J25.

Partially supported by the National Science Foundation Grant DMS-9971009.

(C)2004 American Mathematical Society Reverts to public domain 28 years from publication 
every solution $u$ of the boundary value problem

$$
\left\{\begin{array}{l}
\Delta u=u^{\alpha} \quad \text { in } E \\
u=0 \quad \text { on } \partial E \backslash K
\end{array}\right.
$$

we have

$$
u(x) \leq C(E) \rho(x) \operatorname{dist}(x, K)^{-d} \operatorname{Cap}(K)^{1 /(\alpha-1)} .
$$

1.2. Equivalent definitions of the Poisson capacity. Put

$$
\hat{K} f(y)=\int_{E} f(x) m(d x) k(x, y) .
$$

The following definitions of the Poisson capacity are equivalent to (1.1):

$$
\operatorname{Cap}(K)^{1 / \alpha}=\sup \{\nu(K): \nu \in \mathcal{M}(K), \mathcal{E}(\nu) \leq 1\}
$$

and

$$
\operatorname{Cap}(K)^{1 / \alpha}=\inf \left\{\|f\|_{\alpha^{\prime}}: \hat{K} f \geq 1 \text { on } K\right\}
$$

where $\alpha^{\prime}=\alpha /(\alpha-1)$ and $\|f\|_{\alpha^{\prime}}$ stands for the norm in $L_{\alpha^{\prime}}(m)$.

The equivalence of (1.6) and (1.7) is proved, for instance, in [1] (see Theorem 5.1 in Chapter 13). To prove the equivalence of (1.1) and (1.5), we note that $\nu \in \mathcal{M}(K)$ is equal to $t \mu$ where $t=\nu(K)$ and $\mu=\nu / t \in \mathcal{P}(K)$ and

$$
\begin{aligned}
\sup _{\nu \in \mathcal{M}(K)}\{\nu(K): \mathcal{E}(\nu) \leq 1\} & =\sup _{\mu \in \mathcal{P}(K)} \sup _{t \geq 0}\left\{t: t^{\alpha} \mathcal{E}(\mu) \leq 1\right\} \\
& =\sup _{\mu \in \mathcal{P}(K)} \mathcal{E}(\mu)^{-1 / \alpha}=(\operatorname{Cap}(K))^{1 / \alpha} .
\end{aligned}
$$

1.3. Notation. We denote by $B_{r}(x)$ a ball of radius $r$ centered at $x$. Let $H$ be a compact subset of $\partial E \cap B_{r}(x)$ and let $\phi$ be a $C^{\infty}$ function on $E$ such that $0 \leq \phi \leq 1$. We call $\phi$ an $(R, x)$-truncating function for $H$ if $\phi=0$ in a neighborhood of $H$ and $\phi(y)=1$ if $\operatorname{dist}(x, y) \geq R$. We call $\phi$ an $R$-localizing function if $\phi=0$ in a neighborhood of $H$ and $\phi(y)=1$ if $\operatorname{dist}(y, H) \geq R$.

\section{BOUNDS IN A HALFSPACE}

2.1. First, we establish some bounds in the case when $E=(0, \infty) \times \mathbb{R}^{d-1}$. A generic element of $E$ is denoted by $z$ or $(s, x), s \in(0, \infty), x \in \mathbb{R}^{d-1}$. We use the notation $f(s, x)$ and $f^{s}(x)$ for functions on $E$.

Denote by $\mathbb{E}$ an infinite strip $\{(s, x): 0 \leq s<1\}$. The measure $m(d z)=$ $1_{[0,1]}(s) s d s d x$ is concentrated on $\mathbb{E}$. We denote by $\|f\|_{\alpha}$ the norm in $L_{\alpha}(m)$. The Poisson kernel $k$ can be represented by the formula

$$
k((s, x), y)=C q^{s}(x-y)
$$

where $C$ is a constant depending only on the dimension, and

$$
q^{s}(x)=\frac{s}{\left(|x|^{2}+s^{2}\right)^{d / 2}} .
$$

Lemma 2.1. Suppose that $z_{0} \in \partial E$ and $H$ is a compact subset of $\partial E \cap B_{1}\left(z_{0}\right)$. If $\operatorname{Cap}(H)>0$, then there exists a $\left(3 / 2, z_{0}\right)$-truncating function $\beta$ for $H$ such that $\beta^{s}(x)=1$ for $s \geq 1$ and

$$
\left\|\nabla^{2} \beta\right\|_{\alpha^{\prime}}^{\alpha^{\prime}}+\left\||\nabla \beta|^{2}\right\|_{\alpha^{\prime}}^{\alpha^{\prime}}+\|\nabla \beta\|_{\alpha^{\prime}}^{\alpha^{\prime}}+\left\|\frac{1}{s} \frac{\partial \beta}{\partial s}\right\|_{\alpha^{\prime}}^{\alpha^{\prime}} \leq C(d) \operatorname{Cap}(H)^{1 /(\alpha-1)}
$$


where the constant $C(d)$ depends only on $d$. If $\operatorname{Cap}(H)=0$, then, for every $\epsilon>0$, there exists a $\left(3 / 2, z_{0}\right)$-truncating function $\beta$ for $H$ such that $\beta^{r}(x)=1$ for $r \geq 1$ and

$$
\left\|\nabla^{2} \beta\right\|_{\alpha^{\prime}}^{\alpha^{\prime}}+\left\||\nabla \beta|^{2}\right\|_{\alpha^{\prime}}^{\alpha^{\prime}}+\|\nabla \beta\|_{\alpha^{\prime}}^{\alpha^{\prime}}+\left\|\frac{1}{s} \frac{\partial \beta}{\partial s}\right\|_{\alpha^{\prime}}^{\alpha^{\prime}}<\epsilon .
$$

Proof. Let $\operatorname{Cap}(H)>0$. By (1.7), there exists a function $f$ on $E$ such that $\|f\|_{\alpha^{\prime}}^{\alpha^{\prime}} \leq$ $2 \operatorname{Cap}(H)^{\alpha^{\prime} / \alpha}$ and $\hat{K} f \geq 1$ on $H$. We may assume that $f \geq 0$ (otherwise we just replace $f$ with $\left.f^{+}\right)$.

Let $A(t), 0 \leq t<\infty$, be an increasing $C^{2}$ function such that $A(t)=0$ for $t \leq 1$ and $A(t)=1$ for $t \geq \sqrt{2}$. We set

$$
(\mathcal{T} f)^{t}(y)=\int_{0}^{1} s d s A(\sqrt{s / t}) \int_{\mathbb{R}^{d-1}} f^{s}(x) q^{s}(y-x) d x \quad \text { for } t>0
$$

and

$$
(\mathcal{T} f)^{0}(y)=\lim _{t \downarrow 0}(\mathcal{T} f)^{t}(y)=\hat{K} f(y)
$$

(cf. [1, formula (6.3), p. 175]).

By [1, Theorem 13.6.1], we have

$$
\|\mathcal{T} f\|_{\alpha^{\prime}}+\|\nabla \mathcal{T} f\|_{\alpha^{\prime}}+\left\|\nabla^{2} \mathcal{T} f\right\|_{\alpha^{\prime}}+\left\|\frac{1}{s} \frac{\partial \mathcal{T} f}{\partial s}\right\|_{\alpha^{\prime}} \leq C\|f\|_{\alpha^{\prime}} .
$$

Let $g(s, y)=a(s) b(y)$ be such that $0 \leq a, b \leq 1, a, b$ are $C^{2}$ functions, $a=1$ in a neighborhood of $0, b=1$ in a neighborhood of $\partial E \cap B_{1}\left(z_{0}\right)$ and $g=0$ outside $B_{3 / 2}\left(z_{0}\right)$. Let $h(t)$ be an increasing $C^{2}$ function on $[0, \infty)$ such that $h(t)=0$ if $t \leq 1 / 4$ and $h(t)=1$ if $t \geq 3 / 4$. As in the proof of Lemma 13.6.5 from [1], we put

$$
u=\mathcal{T} f, \quad v=g u, \quad \phi=h(v)
$$

and, finally,

$$
\beta=1-\phi=1-h(g \mathcal{T} f) .
$$

By (2.5), $\mathcal{T} f=\hat{K} f \geq 1$ on $H$, and $\beta=0$ in a neighborhood of $H$ by the choice of $g$ and $h$. By direct computation $\sqrt[1]{1}$ we get

$$
\begin{gathered}
|v|+|\nabla v|+\left|\nabla^{2} v\right| \leq C\left(|u|+|\nabla u|+\left|\nabla^{2} u\right|\right), \\
\left|\frac{1}{s} \frac{\partial v}{\partial s}\right|=\left(\left|\frac{a}{s} \frac{\partial u}{\partial s}+\frac{a^{\prime}}{s} u\right|\right) b \leq C\left(|u|+\frac{1}{s}\left|\frac{\partial u}{\partial s}\right|\right) .
\end{gathered}
$$

More computation yields

$$
\begin{gathered}
|\nabla \phi| \leq C|\nabla v|, \\
\left|\nabla^{2} \phi\right| \leq C\left(\left|\nabla^{2} v\right|+\frac{|\nabla v|^{2}}{v}\right), \\
|\nabla \phi|^{2} \leq C\left(\frac{|\nabla v|^{2}}{v}\right) .
\end{gathered}
$$

Therefore

$$
\left\|\nabla^{2} \beta\right\|_{\alpha^{\prime}}+\left\||\nabla \beta|^{2}\right\|_{\alpha^{\prime}}+\|\nabla \beta\|_{\alpha^{\prime}}+\left\|\frac{1}{s} \frac{\partial \beta}{\partial s}\right\|_{\alpha^{\prime}} \leq C\|f\|_{\alpha^{\prime}} \leq C \operatorname{Cap}(H)^{1 /(\alpha-1)} .
$$

\footnotetext{
${ }^{1}$ See [1, pp. 181-182], or [2] Section 3].
} 
If $\operatorname{Cap}(H)=0$, then $\|f\|_{\alpha^{\prime}}$ can be made arbitrary small, and the same construction yields (2.3).

2.2. For a set $H \subset R^{d-1}$, we put $\lambda H=\{\lambda x: x \in H\}$.

Lemma 2.2. For every compact set $H \subset R^{d-1}$ and every $0<\lambda<1$,

$$
\operatorname{Cap}(\lambda H)^{1 /(\alpha-1)} \leq \lambda^{d-2 \alpha^{\prime}+1} \operatorname{Cap}(H)^{1 /(\alpha-1)} .
$$

Proof. Let $\lambda>0$ and $\nu \in \mathcal{P}(H)$. Then $\nu_{\lambda}(A)=\nu(A / \lambda)$ is concentrated on $\lambda H$. Note that $q^{\lambda s}(\lambda x)=\lambda^{d-1} q^{s}(x)$ and therefore

$$
h_{\nu}(s, x)=\lambda^{d-1} h_{\nu_{\lambda}}(\lambda s, \lambda x) .
$$

Formula (2.12) and change of variables $t=\lambda s, y=\lambda x$ yield

$$
\begin{aligned}
\mathcal{E}(\nu) & =\int_{0}^{1} \int_{\mathbb{R}^{d-1}} h_{\nu}^{\alpha}(s, x) s d s d x \\
& =\int_{0}^{1} \int_{\mathbb{R}^{d-1}} \lambda^{(d-1) \alpha} h_{\nu_{\lambda}}^{\alpha}(\lambda s, \lambda x) s d s d x \\
& =\int_{0}^{\lambda} \int_{\mathbb{R}^{d-1}} \lambda^{(d-1) \alpha} \lambda^{-(d+1)} h_{\nu_{\lambda}}^{\alpha}(t, y) t d t d y \\
& \leq \lambda^{(d-1) \alpha} \lambda^{-(d+1)} \mathcal{E}\left(\nu_{\lambda}\right)
\end{aligned}
$$

and (1.1) implies

$$
\operatorname{Cap}(H) \geq \lambda^{d+1-(d-1) \alpha} \operatorname{Cap}(\lambda H) .
$$

Formula (2.11) follows from (1.1) because $d-2 \alpha^{\prime}+1=-[d+1-(d-1) \alpha] /(\alpha-1)$.

Lemma 2.3. Let $z_{0} \in \partial E, 0<\delta<1$ and let $\Gamma$ be a compact subset of $\partial E \cap B_{\delta}\left(z_{0}\right)$. Suppose $\operatorname{Cap}(\Gamma)>0$. There exists a $\left(3 \delta / 2, z_{0}\right)$-truncating function $\gamma=\gamma_{\Gamma, \delta}$ for $\Gamma$ such that

$$
\left\|\nabla^{2} \gamma\right\|_{\alpha^{\prime}}^{\alpha^{\prime}}+\left\||\nabla \gamma|^{2}\right\|_{\alpha^{\prime}}^{\alpha^{\prime}}+\left\|\frac{1}{\delta} \nabla \gamma\right\|_{\alpha^{\prime}}^{\alpha^{\prime}}+\left\|\frac{1}{s} \frac{\partial \gamma}{\partial s}\right\|_{\alpha^{\prime}}^{\alpha^{\prime}} \leq C(d) \operatorname{Cap}(\Gamma)^{1 / \alpha-1}
$$

where the constant $C(d)$ depends only on $d$. If $\operatorname{Cap}(\Gamma)=0$, then the left side of (2.14) can be made smaller than any $\varepsilon>0$.

Proof. Let $H=\Gamma / \delta$ and let $\beta(s, x)$ be the function constructed in Lemma 2.1 applied to $H$ and $z_{0} / \delta$. Put $\gamma(s, x)=\beta(r / \delta, x / \delta)$. Since $\beta(s, x)=1$ if $s \geq 1$, $\gamma(s, x)=1$ if $s \geq \delta$. Also,

$$
\nabla \gamma(s, x)=(1 / \delta) \nabla \beta(s / \delta, x / \delta), \quad \nabla^{2} \gamma(s, x)=\left(1 / \delta^{2}\right) \nabla^{2} \beta(s / \delta, x / \delta)
$$

and therefore

$$
\begin{aligned}
\|\nabla \gamma\|_{\alpha^{\prime}}^{\alpha^{\prime}} & =\int_{0}^{\delta} \int_{\mathbb{R}^{d-1}}|\nabla \gamma(s, x)|^{\alpha^{\prime}} s d s d x \\
& =\int_{0}^{\delta} \int_{\mathbb{R}^{d-1}} \delta^{-2 \alpha^{\prime}}|\nabla \beta(s / \delta, x / \delta)|^{\alpha^{\prime}} s d s d x \\
& =\int_{0}^{1} \int_{\mathbb{R}^{d-1}} \delta^{d+1-2 \alpha^{\prime}}|\nabla \beta(s, x)|^{\alpha^{\prime}} s d s d x=\delta^{d+1-2 \alpha^{\prime}}\|\beta\|_{\alpha^{\prime}}^{\alpha^{\prime}}
\end{aligned}
$$


In a similar way,

$$
\left\||\nabla \gamma|^{2}\right\|_{\alpha^{\prime}}^{\alpha^{\prime}}=\delta^{d+1-2 \alpha^{\prime}}\left\||\nabla \beta|^{2}\right\|_{\alpha^{\prime}}^{\alpha^{\prime},} \quad\left\|\frac{1}{\delta} \nabla \gamma\right\|_{\alpha^{\prime}}^{\alpha^{\prime}}=\delta^{d+1-2 \alpha^{\prime}}\|\nabla \beta\|_{\alpha^{\prime}}^{\alpha^{\prime}}
$$

and

$$
\left\|\frac{1}{s} \frac{\partial \gamma}{\partial s}\right\|_{\alpha^{\prime}}^{\alpha^{\prime}}=\left\|\frac{1}{s} \frac{\partial \beta}{\partial s}\right\|_{\alpha^{\prime}}^{\alpha^{\prime}} .
$$

Therefore (2.14) follows from (2.2) and Lemma 2.2.

\section{BOUNDS IN A UNIT BALL}

3.1. Now let $E$ be a ball of radius 1 in $\mathbb{R}^{d}$ centered at a point $z_{0}$ with coordinates $s=1, x=0$. As before, let $\mathbb{E}=\{(s, x): 0 \leq s<1\}$. For a point $z=(s, x) \in \mathbb{E}$, we denote by

$$
\phi(z)=x /(1-s)
$$

a projection of $z$ to $\mathbb{R}^{d-1}$ with center at $z_{0}$. For a point $z \in E \cap \mathbb{E}$, we put

$$
\psi(z)=\left(1-\left|z-z_{0}\right|, \phi(z)\right)
$$

(cf. [3], Section 3.1.1). The mapping $\psi$ defines a 1-1 correspondence between $E \cap \mathbb{E}$ and $\mathbb{E}$.

For a set $H \subset \partial E$, denote by $\operatorname{Cap}_{E}(H)$ the Poisson capacity of $H$ with respect to the domain $E$ and the measure $m(d z)=\operatorname{dist}(z, \partial E) d z$. For a set $K \subset \mathbb{R}^{d-1}$, we denote by $\operatorname{Cap}_{\mathbb{E}}(K)$ the Poisson capacity with respect to the halfspace and the measure $m(d s, d x)=1_{[0,1)}(s) s d s d x$.

Lemma 3.1. Let $H$ be a compact subset of $\partial E$ that is contained in a ball of radius $1 / 4$ centered at zero, and let $K=\psi(H)$. There exists a constant $C$ depending only on the dimension, such that

$$
C^{-1} \operatorname{Cap}_{\mathbb{E}}(K) \leq \operatorname{Cap}_{E}(H) \leq C \operatorname{Cap}_{\mathbb{E}}(K) .
$$

Proof. Let $\mu$ be a probability measure on $H$ and let $\nu$ be a measure on $K$ defined by the formula $\nu(\Gamma)=\mu\left(\psi^{-1}(\Gamma)\right)$. It is enough to show that

$$
C^{-1} \mathcal{E}_{E}(\mu)<\mathcal{E}_{\mathbb{E}}(\nu)<C \mathcal{E}_{E}(\mu)
$$

for some constant $C$ depending only on the dimension.

Denote by $D$ a ball of radius $1 / 2$ centered at zero. Let $D^{\prime}=\psi(D)$. Put

$$
I_{E}=\int_{E \backslash D} \rho(z) h_{\mu}^{\alpha}(z) d z, \quad J_{E}=\int_{E \cap D} \rho(x) h_{\mu}^{\alpha}(x) d x
$$

and

Note that

$$
I_{\mathbb{E}}=\int_{\mathbb{E} \backslash D^{\prime}} \rho(z) h_{\nu}^{\alpha}(z) d z, \quad J_{\mathbb{E}}=\int_{\mathbb{E} \cap D^{\prime}} \rho(z) h_{\nu}^{\alpha}(z) d z .
$$

$$
C^{-1} \rho(z) /(|z|+1 / 4)^{d}<h_{\mu}(z) \leq C \rho(z) /(|z|-1 / 4)^{d}
$$

on $E \backslash D$ and therefore $C^{-1}<I_{E}<C$. For the same reason, $C^{-1}<I_{\mathbb{E}}<C$ and therefore

$$
C^{-1} I_{E}<I_{\mathbb{E}}<C I_{E} .
$$

On the other hand,

$$
C^{-1} h_{\nu}(\psi(z))<h_{\mu}(z)<C h_{\nu}(\psi(z))
$$


on $D$ (this follows from a similar relation for the Poisson kernels). Since the derivatives of $\psi$ and $\psi^{-1}$ are bounded on $D$ and $D^{\prime}$, we conclude that

$$
C^{-1} J_{E}<J_{\mathbb{E}}<C J_{E} .
$$

Since $\mathcal{E}_{E}(\mu)=I_{E}+J_{E}$ and $\mathcal{E}_{\mathbb{E}}(\nu)=I_{\mathbb{E}}+J_{\mathbb{E}}$, (3.1) follows from (3.2) and (3.3).

Lemma 3.2. Let $H$ be a compact subset of $\partial E$ such that $H \subset B_{\delta / 8}(0)$, where $\delta \leq 2$. Suppose $\operatorname{Cap}_{E}(H)>0$. There exists a $(3 \delta / 8,0)$-truncating function $\gamma$ such that

$$
\left\|\nabla^{2} \gamma\right\|_{\alpha^{\prime}}^{\alpha^{\prime}}+\left\||\nabla \gamma|^{2}\right\|_{\alpha^{\prime}}^{\alpha^{\prime}}+\left\|\frac{1}{\delta} \nabla \gamma\right\|_{\alpha^{\prime}}^{\alpha^{\prime}}+\left\|\frac{1}{\rho} \frac{\partial \gamma}{\partial \rho}\right\|_{\alpha^{\prime}}^{\alpha^{\prime}} \leq C(d) \operatorname{Cap}_{E}(H)^{1 /(\alpha-1)},
$$

where the constant $C(d)$ depends only on $d$. If $\operatorname{Cap}_{E}(H)=0$, then the left side of (3.4) can be made smaller than any $\varepsilon>0$.

Proof. We apply Lemma 2.3 to the set $K=\psi(H)$. Let $\gamma_{K}$ be the function constructed in Lemma 2.3. We put $\gamma(s, x)=\gamma_{K}(\psi(s, x))$ if $s<1$, and $\gamma(s, x)=0$ otherwise. Similarly to the proof of [3, Sublemma 3.1.2], we show that

$$
\begin{aligned}
\left\|\nabla^{2} \gamma\right\|_{E, \alpha^{\prime}}^{\alpha^{\prime}} & +\left\|\left.\nabla \gamma\right|^{2}\right\|_{E, \alpha^{\prime}}^{\alpha^{\prime}}+\left\|\frac{1}{\delta} \nabla \gamma\right\|_{E, \alpha^{\prime}}^{\alpha^{\prime}}+\left\|\frac{1}{\rho} \frac{\partial \gamma}{\partial \rho}\right\|_{E, \alpha^{\prime}}^{\alpha^{\prime}} \\
& \leq\left\|\nabla^{2} \gamma_{K}\right\|_{\mathbb{E}, \alpha^{\prime}}^{\alpha^{\prime}}+\left\|\left|\nabla \gamma_{K}\right|^{2}\right\|_{\mathbb{E}, \alpha^{\prime}}^{\alpha^{\prime}}+\left\|\frac{1}{\delta} \nabla \gamma_{K}\right\|_{\mathbb{E}, \alpha^{\prime}}^{\alpha^{\prime}}+\left\|\frac{1}{s} \frac{\partial \gamma_{K}}{\partial s}\right\|_{\mathbb{E}, \alpha^{\prime}}^{\alpha^{\prime}}
\end{aligned}
$$

where $\|\cdot\|_{E}$ and $\|\cdot\|_{\mathbb{E}}$ stand for $L_{\alpha^{\prime}}$-norms in $E$ and $\mathbb{E}$. Finally, we apply Lemma 3.1.

3.2. Localizing functions. Let $H$ be a subset of $\partial E$ and let $\gamma$ be a $C^{2}$-function on $E$. We call $\gamma$ an $\varepsilon$-localizing function for $H$ if $0 \leq \gamma \leq 1, \gamma=1$ in a neighborhood of $H$ and $\gamma(z)=0$ if $\operatorname{dist}(z, \mathcal{H})>\varepsilon$.

Lemma 3.3. There exists a constant $C(d)$ such that, for every compact subset $K$ of $\partial E$ with $\operatorname{Cap}(K)>0$ and $\operatorname{diam}(K) \leq 4 \delta$, there exists a $\delta / 2$-localizing function $\gamma=\gamma_{\delta, K}$ for $K$ such that

$$
\left\|\nabla^{2} \gamma\right\|_{\alpha^{\prime}}^{\alpha^{\prime}}+\left\||\nabla \gamma|^{2}\right\|_{\alpha^{\prime}}^{\alpha^{\prime}}+\left\|\frac{1}{\delta} \nabla \gamma\right\|_{\alpha^{\prime}}^{\alpha^{\prime}}+\left\|\frac{1}{\rho} \frac{\partial \gamma}{\partial \rho}\right\|_{\alpha^{\prime}}^{\alpha^{\prime}} \leq C(d) \operatorname{Cap}(K)^{1 /(\alpha-1)} .
$$

Proof. As in [3, Lemma 3.1.2], we cover the set $K$ by finitely many balls $B_{\delta / 8}\left(y_{k}\right)$ (the number $n$ of the balls depends only on the dimension $d$ ). We apply Lemma 3.2 to each of the sets $H_{k}=K \cap B_{\delta / 8}\left(y_{k}\right)$. Denote by $\gamma_{k}$ the corresponding truncating function constructed in Lemma 3.2 (we choose $\epsilon=\operatorname{Cap}(\Gamma)^{1 /(\alpha-1)}$ if $\operatorname{Cap}\left(H_{k}\right)=0$ for some $k$ ). We set

$$
\gamma=\gamma_{1} \cdots \gamma_{n} .
$$

Note that

$$
|\nabla \gamma| \leq \sum_{k}\left|\nabla \gamma_{k}\right|
$$


and

$$
\begin{aligned}
\left|\nabla^{2} \gamma\right| & \leq \sum_{k}\left|\nabla^{2} \gamma_{k}\right|+\sum_{k \neq l}\left|\nabla \gamma_{k}\right|\left|\nabla \gamma_{l}\right| \\
& \leq \sum_{k}\left|\nabla^{2} \gamma_{k}\right|+\frac{(n-1)}{2} \sum_{k}\left|\nabla \gamma_{k}\right|^{2} .
\end{aligned}
$$

By applying Minkowski inequality, we get

$$
\begin{aligned}
\left\|\nabla^{2} \gamma\right\|_{\alpha^{\prime}}^{\alpha^{\prime}} & +\left\||\nabla \gamma|^{2}\right\|_{\alpha^{\prime}}^{\alpha^{\prime}}+\left\|\frac{1}{\delta} \nabla \gamma\right\|_{\alpha^{\prime}}^{\alpha^{\prime}}+\left\|\frac{1}{\rho} \frac{\partial \gamma}{\partial \rho}\right\|_{\alpha^{\prime}}^{\alpha^{\prime}} \\
& \leq C(d, n) \sum_{k} \operatorname{Cap}\left(H_{k}\right)^{1 /(\alpha-1)}+n C(n) \epsilon \\
& \leq n(C(d, n)+C(n)) \operatorname{Cap}(\Gamma)^{1 /(\alpha-1)} .
\end{aligned}
$$

Lemma 3.4. Let $K$ be a compact subset of $\partial E$ such that $\operatorname{diam}(K) \leq 4 \delta$ and $\operatorname{Cap}(K)>0$. Let $\gamma$ be the $\delta / 2$-localizing function constructed in Lemma 3.3. Then

$$
\begin{aligned}
& \int_{E} u \gamma^{2 \alpha^{\prime}-1}|\Delta \gamma| \rho \leq C\left(\int_{E} u^{\alpha} \gamma^{2 \alpha^{\prime}} \rho\right)^{1 / \alpha} \operatorname{Cap}(K)^{1 / \alpha}, \\
& \int_{E} u \gamma^{2 \alpha^{\prime}-2}|\nabla \gamma|^{2} \rho \leq C\left(\int_{E} u^{\alpha} \gamma^{2 \alpha^{\prime}} \rho\right)^{1 / \alpha} \operatorname{Cap}(K)^{1 / \alpha}, \\
& \int_{E} u \gamma^{2 \alpha^{\prime}-1}|\nabla \gamma| \rho \leq C\left(\int_{E} u^{\alpha} \gamma^{2 \alpha^{\prime}} \rho\right)^{1 / \alpha} \delta \operatorname{Cap}(K)^{1 / \alpha}, \\
& \int_{E} u \gamma^{2 \alpha^{\prime}-1}\left|\frac{\partial \gamma}{\partial \rho}\right| \leq C\left(\int_{E} u^{\alpha} \gamma^{2 \alpha^{\prime}} \rho\right)^{1 / \alpha} \operatorname{Cap}(K)^{1 / \alpha}
\end{aligned}
$$

whenever $u$ satisfies (1.3).

Proof. The assertion follows from the Hölder inequality, Lemma 3.3, the identity $(\alpha-1) \alpha^{\prime}=\alpha$ and the inequality $\gamma^{\left(2 \alpha^{\prime}-1\right) \alpha} \leq \gamma^{\left(2 \alpha^{\prime}-2\right) \alpha}=\gamma^{2 \alpha^{\prime}}$. For instance, for the last line in (3.8), we have

$$
\begin{aligned}
\int_{E} u \gamma^{2 \alpha^{\prime}-1}\left|\frac{\partial \gamma}{\partial \rho}\right| & =\int_{E} u \gamma^{2 \alpha^{\prime}-1}\left|\frac{1}{\rho} \frac{\partial \gamma}{\partial \rho}\right| \rho \\
& \leq\left(\int_{E} u^{\alpha} \gamma^{\left(2 \alpha^{\prime}-1\right) \alpha} \rho\right)^{1 / \alpha}\left(\int_{E}\left|\frac{1}{\rho} \frac{\partial \gamma}{\partial \rho}\right|^{\alpha^{\prime}} \rho\right)^{1 / \alpha^{\prime}} \\
& \leq\left(\int_{E} u^{\alpha} \gamma^{2 \alpha^{\prime}} \rho\right)^{1 / \alpha} C(d) \operatorname{Cap}(K)^{1 /\left[\alpha^{\prime}(\alpha-1)\right]}
\end{aligned}
$$

Lemma 3.5. Let $K, \gamma, u$ be as in Lemma 3.4. There exists a constant $C(d)$ such that

$$
\int_{E} u^{\alpha} \gamma^{2 \alpha^{\prime}} \rho \leq C(d) \operatorname{Cap}(K)^{1 /(\alpha-1)}
$$

whenever u satisfies (1.3). 
Proof. This is an adaptation of Lemma 3.1.3 in [3]. Let $E_{s}=B\left(s, z_{0}\right)$ and $r=$ $\left|z-z_{0}\right|$. By replacing $u^{2}$ and $\gamma^{4}$ with $u^{\alpha}$ and $\gamma^{2 \alpha^{\prime}}$ in the arguments of [3], we get a bound

$$
\begin{aligned}
\int_{E} u^{\alpha} \gamma^{2 \alpha^{\prime}} \rho \leq & \int_{E} u \Delta\left(\gamma^{2 \alpha^{\prime}}\left(1-r^{2}\right)\right)-4 \int_{E} u \frac{\partial\left(\gamma^{2 \alpha^{\prime}}\right)}{\partial r} r \\
& +\liminf _{s \rightarrow 1-} \int_{\partial E_{s}} \frac{\partial}{\partial r}\left(u \gamma^{2 \alpha^{\prime}}\left(1-r^{2}\right)\right) .
\end{aligned}
$$

As in [3, Sublemma 3.1.3], one can show that the last term in (3.10) is negative and can be dropped.

Finally, we note that $1-r^{2} \leq 2 \rho$ and therefore

$$
\begin{aligned}
\mid \int_{E} u & \Delta\left(\gamma^{2 \alpha^{\prime}}\left(1-r^{2}\right)\right) \mid \\
& \leq 2 \alpha^{\prime} \int_{E} u \gamma^{2 \alpha^{\prime}-1}|\Delta \gamma|\left(1-r^{2}\right)+2 \alpha^{\prime}\left(2 \alpha^{\prime}-1\right) \int_{E} u \gamma^{2 \alpha^{\prime}-2}|\nabla \gamma|^{2} \rho \\
& \leq 4 \alpha^{\prime} \int_{E} u \gamma^{2 \alpha^{\prime}-1}|\Delta \gamma|\left(1-r^{2}\right)+4 \alpha^{\prime}\left(2 \alpha^{\prime}-1\right) \int_{E} u \gamma^{2 \alpha^{\prime}-2}|\nabla \gamma|^{2} \rho \\
& \leq C\left(\int_{E} u{ }^{\alpha} \gamma^{2 \alpha^{\prime}} \rho\right)^{1 / \alpha} \operatorname{Cap}(K)^{1 / \alpha} .
\end{aligned}
$$

In a similar way,

$$
\begin{aligned}
\left|\int_{E} u \frac{\partial\left(\gamma^{2 \alpha^{\prime}}\right)}{\partial r} r\right| & =2 \alpha^{\prime}\left|\int_{E} u \gamma^{2 \alpha^{\prime}-1} \frac{\partial \gamma}{\partial r} r\right| \leq 2 \alpha^{\prime}\left|\int_{E} u \gamma^{2 \alpha^{\prime}-1} \frac{\partial \gamma}{\partial r}\right| \\
& \leq C\left(\int_{E} u^{\alpha} \gamma^{2 \alpha^{\prime}} \rho\right)^{1 / \alpha} \operatorname{Cap}(K)^{1 / \alpha} .
\end{aligned}
$$

From (3.10), (3.11) and (3.12), we get

$$
\int_{E} u^{\alpha} \gamma^{2 \alpha^{\prime}} \rho \leq C\left(\int_{E} u^{\alpha} \gamma^{2 \alpha^{\prime}} \rho\right)^{1 / \alpha} \operatorname{Cap}(K)^{1 / \alpha},
$$

which implies (3.9).

Combining (3.8) with Lemma 3.5, we get:

Lemma 3.6. Let $K, \gamma, u$ be as in Lemma 3.4. Then

$$
\begin{gathered}
\int_{E} u \gamma^{2 \alpha^{\prime}-1}|\Delta \gamma| \rho \leq C \operatorname{Cap}(K)^{1 /(\alpha-1)} \\
\int_{E} u \gamma^{2 \alpha^{\prime}-2}|\nabla \gamma|^{2} \rho \leq C \operatorname{Cap}(K)^{1 /(\alpha-1)}, \\
\int_{E} u \gamma^{2 \alpha^{\prime}-1}|\nabla \gamma| \rho \leq C \delta \operatorname{Cap}(K)^{1 /(\alpha-1)}, \\
\int_{E} u \gamma^{2 \alpha^{\prime}-1}\left|\frac{\partial \gamma}{\partial \rho}\right| \leq C \operatorname{Cap}(K)^{1 /(\alpha-1)}
\end{gathered}
$$


3.3. The rest of the proof of Theorem 1.1 is very close to the corresponding part of the proof of [3, Theorem 3.1.1]. We begin with

Lemma 3.7. Let $K, \gamma, u$ be as in Lemma 3.4, and let $G_{E}$ be the Green operator of E. For every $y \in E, \beta>0$,

$$
\gamma^{\beta}(y) u(y) \leq \frac{1}{2} G_{E}\left(\gamma^{\beta} \Delta u-\Delta\left(\gamma^{\beta} u\right)\right)(y) .
$$

This is a version of [3, Lemma 3.1.6] (in [3], $\beta=4$ ). Bounds from [3, Sublemma 3.1.5] must be replaced with those of [1, Theorem 7.1]. Other modifications are obvious.

As a first step, we establish

Lemma 3.8. There exists a constant $C(d)$ such that the inequality (1.4) holds whenever

$$
\operatorname{dist}(x, K) \geq \frac{\operatorname{diam}(K)}{4}
$$

Proof. The proof is an appropriate modification of the proof of [3, Lemma 3.1.5]. Let $\delta=\operatorname{dist}(x, K)$ and let $\gamma$ be the $\delta / 2$-localizing function constructed in Lemma 3.3. Clearly, $\gamma=1$ in a neighborhood of $x$ and therefore

$$
u(x)=\gamma^{2 \alpha^{\prime}}(x) u(x) \leq \frac{1}{2} G_{E}\left(\gamma^{2 \alpha^{\prime}} \Delta u-\Delta\left(\gamma^{2 \alpha^{\prime}} u\right)\right)(x)
$$

by Lemma 3.7 As in [3], the right side can be evaluated by means of Green's formula applied to the domain $B\left(z_{0}, r\right) \backslash B(x, \varepsilon)$ and passage to the limit as $\varepsilon \rightarrow 0, r \rightarrow 1$. Namely, we get

$$
\begin{aligned}
G_{E}\left(\gamma^{2 \alpha^{\prime}} \Delta u\right. & \left.-\Delta\left(\gamma^{2 \alpha^{\prime}} u\right)\right)(x) \\
& =\int_{E}\left(2 \nabla_{y} g_{E}(x, y) \nabla\left(\gamma^{2 \alpha^{\prime}}(y)-g_{E}(x, y) \Delta\left(\gamma^{2 \alpha^{\prime}}\right)(y)\right) u(y) d y\right) .
\end{aligned}
$$

Together with (3.14), this implies

$$
\begin{aligned}
u(x) \leq & 2 \alpha^{\prime} \int_{E} u(y) \gamma^{2 \alpha^{\prime}-1}(y) \nabla \gamma(y) \nabla_{y} g_{E}(x, y) d y \\
& +\alpha^{\prime} \int_{E} u(y) \gamma^{2 \alpha^{\prime}-1}(y) \Delta \gamma(y) g_{E}(x, y) d y \\
& +\alpha^{\prime}\left(2 \alpha^{\prime}-1\right) \int_{E} u(y) \gamma^{2 \alpha^{\prime}-2}(y)|\nabla \gamma(y)|^{2} g_{E}(x, y) d y
\end{aligned}
$$

(cf. 3. (3.13)]). Now, $\gamma(y)=1$ for all $y$ such that $\operatorname{dist}(y, K)>\delta / 2$, in particular for all $y$ such that $|x-y|<\delta / 2$, and therefore the integrands are equal to 0 for such $y$. Following [3], from the bounds for the Green's function and its gradient, we get bounds for the integrals on the right side of (3.15) in terms of integrals (3.13). For instance,

$$
g_{E}(x, y) \leq C \rho(x) \rho(y)|x-y|^{-d},
$$


and therefore

$$
\begin{aligned}
\int_{E} u & (y) \gamma^{2 \alpha^{\prime}-2}(y)|\nabla \gamma(y)|^{2} g_{E}(x, y) d y \\
& \leq C \rho(x) \int_{E} u(y) \gamma^{2 \alpha^{\prime}-2}(y)|\nabla \gamma(y)|^{2} \rho(y)|x-y|^{-d} d y \\
& =\int_{E \backslash B(x, \delta / 2)} u(y) \gamma^{2 \alpha^{\prime}-2}(y)|\nabla \gamma(y)|^{2} \rho(y)|x-y|^{-d} d y \\
& \leq C \rho(x) \delta^{-d} \int_{E} u \gamma^{2 \alpha^{\prime}-2}(y)|\nabla \gamma|^{2}(y) \rho(y) d y .
\end{aligned}
$$

In a similar way, we get

$$
\begin{aligned}
& \int_{E} u(y) \gamma^{2 \alpha^{\prime}-1}(y) \nabla \gamma(y) \nabla_{y} g_{E}(x, y) d y \\
& \quad \leq C \rho(x) \delta^{-d} \int_{E} u \gamma^{2 \alpha^{\prime}-1}\left|\frac{\partial \gamma}{\partial \rho}\right|+C \rho(x) \delta^{-d-1} \int_{E} u \gamma^{2 \alpha^{\prime}-1}|\nabla \gamma| \rho, \\
& \int_{E} u(y) \gamma^{2 \alpha^{\prime}-1}(y) \Delta \gamma(y) g_{E}(x, y) d y \leq C \rho(x) \delta^{-d} \int_{E} u \gamma^{2 \alpha^{\prime}-1}|\Delta \gamma| \rho .
\end{aligned}
$$

It remains to use the bounds of Lemma 3.6.

Theorem 1.1 can be derived from this by using the same construction as in [3]. Let $x \in E$ and $K, u$ be as in Theorem 1.1. Let $\delta=\operatorname{dist}(x, K)$. We set

$$
K_{1}=K \cap \overline{B(x, 2 \delta)},
$$

and

$$
K_{n}=K \cap\left(\overline{B\left(x, 2^{n} \delta\right)} \backslash B\left(x, 2^{n-1} \delta\right)\right), \quad n \geq 2 .
$$

Since $K=\bigcap K_{n}$, we have

$$
u \leq u_{K} \leq \sum u_{K_{n}}
$$

where $u_{K}$ stands for the maximal solution of problem (1.3). By construction, we have $\operatorname{dist}\left(x, K_{n}\right) \geq 2^{n-1} \delta$ and $\operatorname{diam}\left(K_{n}\right) \leq 2^{n+1} \delta$. Therefore Lemma 3.8 is applicable to every $K_{n}$ and we get

$$
u_{K_{n}} \leq C \rho(x) 2^{-n d} \delta^{-d} \operatorname{Cap}\left(K_{n}\right)^{1 /(\alpha-1)} \leq C \rho(x) 2^{-n d} \delta^{-d} \operatorname{Cap}(K)^{1 /(\alpha-1)},
$$

which implies

$$
u(x) \leq \sum u_{K_{n}} \leq C \rho(x) \delta^{-d} \operatorname{Cap}(K)^{1 /(\alpha-1)} \sum 2^{-n d} .
$$

The extension of the theorem to arbitrary $C^{4}$ domains is a simple modification of the arguments by Mselati 3 .

\section{REFERENCES}

1. E. B. Dynkin, Diffusions, superdiffusions and partial differential equations, American Mathematical Society, Providence, RI, 2002. MR.1883198|(2003c:60001)

2. S. E. Kuznetsov, Polar boundary sets for superdiffusions and removable lateral singularities for nonlinear parabolic PDEs, Comm. Pure Appl. Math. 51 (1998), 303-340. MR1488517 (99c:35111)

3. B. Mselati, Classification et représentation probabiliste des solutions positives de $\Delta u=u^{2}$ dans un domaine, Thése de Doctorat de l'Université Paris 6, 2002.

Department of Mathematics, University of Colorado, Boulder, CO 80309-0395

E-mail address: Sergei.Kuznetsov@Colorado.edu 